\title{
Tubercular Mastitis Masquerading as Carcinoma Breast in Elderly Female: A Diagnostic Dilemma
}

\begin{abstract}
Tubercular mastitis (TM), a great masquerader, is extremely rare in elderly. Diagnosis becomes challenging when patient's age and clinical features favor carcinoma, as in our case, where a 70 -year-old female presented with right breast lumps associated with overlying ulcers and right supraclavicular lymphadenopathy. Fine-needle aspiration cytology of breast lump and lymph node followed by special staining and culture confirmed TM and lymphadenitis. Antitubercular therapy was initiated, and the patient got prompt relief. Here, we have discussed clinicocytological aspects of TM mimicking as carcinoma breast and diagnostic approach that should be undertaken. The case highlights the value of precise diagnosis, as specific treatment is indicated for tubercular and other granulomatous mastitis; placing an emphasis on meticulous investigation to rule out possibility of lethal malignancy in elderly; and necessity to always keep possibility of even rare entity like TM, irrespective of age, especially in India, where tuberculosis is endemic.
\end{abstract}

Keywords: Breast, carcinoma, elderly, fine-needle aspiration cytology, granulomatous, mastitis, tubercular

\section{Introduction}

Tuberculosis is highly endemic in India, but still, breast tuberculosis is extremely rare. Incidence ranges from $0.1 \%$ in developed countries to $4 \%$ in highly endemic countries like India. $^{[1]}$ Tubercular mastitis (TM) is extremely rare in elderly females, ${ }^{[2]}$ adolescent females and males. It affects young females of reproductive age 20-40 years, associated risk factors being multiparity, lactation, ${ }^{[3]}$ trauma, suppurative mastitis, and immunocompromised states. ${ }^{[4]}$ The causative agent Mycobacterium tuberculosis can infect multiple organs resulting in disseminated extrapulmonary tuberculosis. However, spleen, muscle, and breast confer resistance to multiplication of bacilli, and hence its survival. ${ }^{[4]}$ Our case with clinical suspicion of carcinoma breast throws light on how to approach such patients, to avoid erroneous diagnosis and grievous consequences.

\section{Case Report}

A 70-year-old Indian woman presented with right breast lumps for 2 months. On examination, multiple, ill-defined, firm-to-hard, nontender right breast lumps were noted involving upper outer quadrant,

This is an open access journal, and articles are distributed under the terms of the Creative Commons Attribution-NonCommercial-ShareAlike 4.0 License, which allows others to remix, tweak, and build upon the work non-commercially, as long as appropriate credit is given and the new creations are licensed under the identical terms. ranging in size from 2 to $4 \mathrm{~cm}$ in maximum dimension, associated with indurated and ulcerated overlying skin, right nipple retraction, and enlarged right supraclavicular lymph node measuring $2 \mathrm{~cm}$ in diameter. She denied any history of nipple discharge, fever, weight loss, or systemic symptoms. Her past and family histories were unremarkable. Her provisional clinical diagnosis was carcinoma breast with metastasis to right supraclavicular lymph node. She was advised breast imaging and fine-needle aspiration cytology (FNAC) of breast lumps and right supraclavicular lymph node. Her breast imaging findings raised the possibility of breast abscess or carcinoma. Subsequently, after obtaining written informed consent from her, she underwent FNAC in our department. Minimum three slides each for May-Grunwald-Giemsa (MGG) and Papanicolaou's stain (PAP) were prepared. The cheesy aspirate obtained from right supraclavicular lymph node prompted us for Ziehl-Neelsen (ZN) staining.

Cytology smears from breast lumps and lymph node revealed multiple granulomas [Figure 1a and b] comprised of epithelioid histiocytes, lymphocytes, and macrophages against large areas of necrosis [Figure 2]. ZN stain revealed classical

\footnotetext{
How to cite this article: Das $A$, Panwar $\mathrm{H}$, Hussain N. Tubercular mastitis masquerading as carcinoma breast in elderly female: $A$ diagnostic dilemma. Indian J Med Paediatr Oncol 2018;39:516-8.
}

\section{Aditi Das, Hemlata Panwar, Nighat Hussain}

Department of Pathology and Lab Medicine, AIIMS, Raipur, Chhattisgarh, India
Address for correspondence: Dr. Hemlata Panwar, Department of Pathology and Lab Medicine, AIIMS, Raipur, Chhattisgarh, India. E-mail: hemlatasongra@gmail. com

\section{Access this article online}

Website: www.ijmpo.org

DOI: 10.4103/ijmpo.ijmpo_103_17 Quick Response Code:

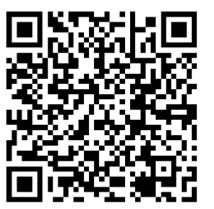




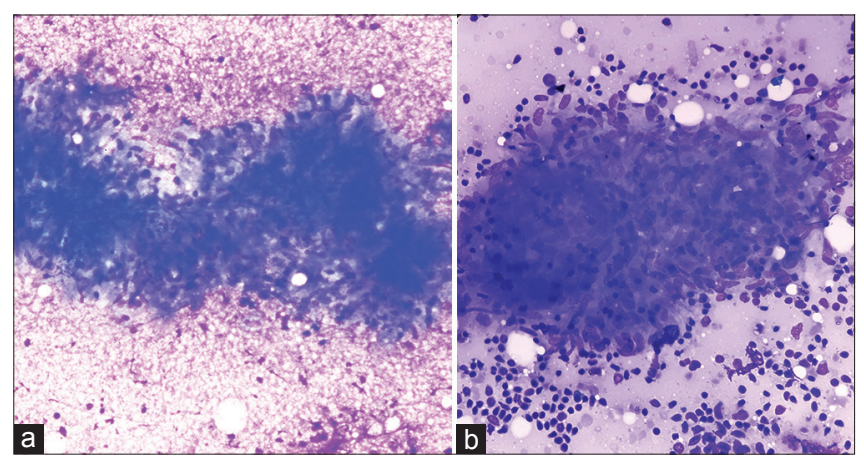

Figure 1: (a) Photomicrograph of breast lump aspiration smear displaying classical granuloma comprised of epithelioid histiocytes and lymphocytes on necrotic background (MGG, $\times 400$ ). (b) Photomicrograph of supraclavicular lymph node aspiration smear displaying cluster of loosely cohesive epithelioid histiocytes with characteristic elongated sole-shaped nuclei; along with lymphocytes and lymphoglandular bodies on background (MGG, $\times 400$ )

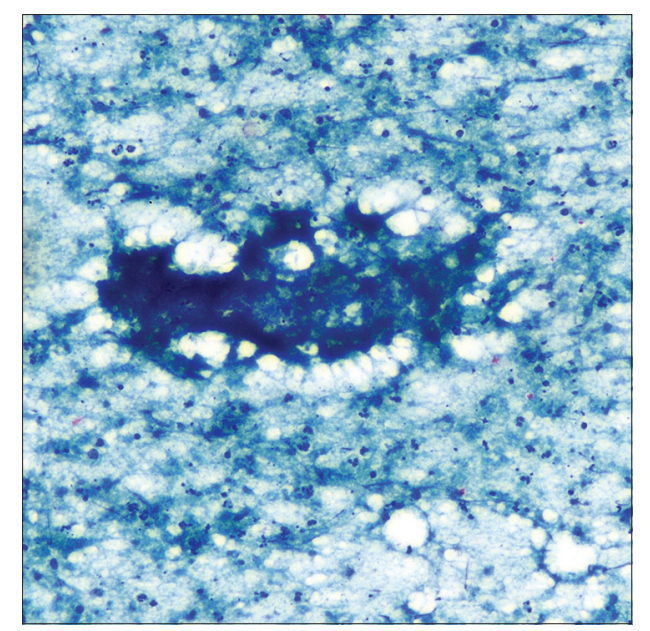

Figure 2: Photomicrograph of breast lump aspiration smear displaying ill-defined granuloma on granular caseous necrotic background (PAP, $\times 100)$

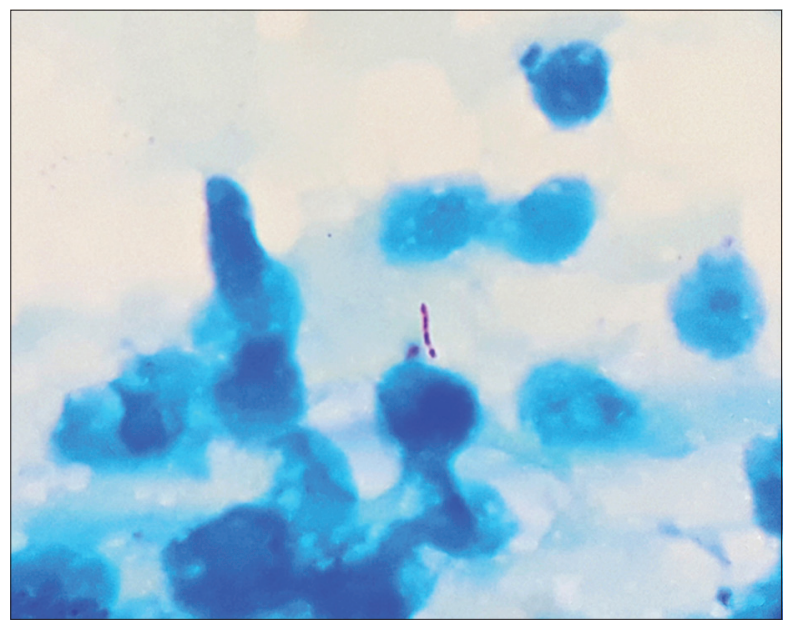

Figure 3: Photomicrograph displaying classical "beaded," pink-colored, "rod-shaped," acid-fast bacilli (Ziehl-Neelsen, ×100)

acid-fast bacilli (AFB) [Figure 3]. Cytological diagnosis was suggestive of TM and lymphadenitis; subsequently, culture confirmed the bacilli as M. tuberculosis. Chest imaging performed later, was unremarkable, and did not suggest current or previous tubercular infection. Six months course of Directly Observed Therapy Short-Term (DOTS) - Category I comprising standard four-drug anti-tubercular therapy (ATT) was initiated and ulcer debridement with abscess drainage was done. On follow-up, breast lumps were not palpable, and the local wound got healed satisfactorily.

\section{Discussion}

In elderly females, breast carcinoma is much common than TM. In such females, TM poses a diagnostic challenge, as it is extremely rare entity mimicking carcinoma breast. Irregular hard breast lumps, especially if fixed to overlying skin, underlying muscle or chest wall, associated with overlying skin ulcers and/or lymphadenopathy suggest malignancy. TM also usually presents with solitary painful breast lump associated with overlying skin inflammation, ulcers, draining sinuses, and abscess. ${ }^{[5]}$ Constitutional symptoms though less common, but when present usually favors inflammatory lesion like TM, especially, if associated with intact nipple and areola and/or draining sinuses in young, multiparous, or lactating females. ${ }^{[4]}$ Primary TM without primary foci can be caused through duct openings in the nipple or through skin abrasions. In secondary forms, major routes of spread are lymphatic and/or hematogenous from pulmonary or distant extrapulmonary foci and contiguous spread from pleura and chest wall. ${ }^{[4]}$

The proposed newer classification of TM is: ${ }^{[4]}$

i. Nodulocaseous - Most common; well-circumscribed, slow-growing painless mass, may involve overlying skin, ulcerate, form sinuses and may become painful, mimicking fibroadenoma in early stages while carcinoma in later stages

ii. Disseminated/confluent - Multiple caseating foci leading to sinus formation with/without overlying painful ulcers associated with enlarged draining axillary lymph nodes

iii. Abscess - Common in young females.

The important differential diagnoses of TM such as locally advanced breast cancer (LABC) and inflammatory breast carcinoma (IBC) should be ruled out by thorough investigations. Currently, LABC refers to most advanced breast cancer without distant metastases. ${ }^{[6]}$ Definition of LABC according to the recent guidelines from the US National Comprehensive Cancer Network ${ }^{[6]}$ has been summarized in Table 1.

IBC is very aggressive and lethal subtype of LABC. Diagnosis is based on clinical features such as rapid-onset diffuse edema (peau d' orange-the hallmark of $I B C$ ) and erythema over breast skin, with or without an underlying palpable mass. Inflammation actually results from dermal lymphatics impaction by tumor emboli. ${ }^{[7]}$ Pathological evaluation is absolutely necessary to differentiate among carcinomas, granulomatous mastitis and TM. 
Table 1: Recent guidelines from US National Comprehensive Cancer Network states locally advanced breast cancer as American Joint Cancer Committee Stage III breast cancer and the definition includes any of the following criteria in the absence of distant metastasis

\begin{tabular}{lll}
\hline Tumor size & Regional lymphadenopathy & Special comment \\
\hline $5 \mathrm{~cm}$ or & N1-N3 & -
\end{tabular}
more Any size Present or absent

Tumor directly extending to chest wall or skin, or both (including ulcer or satellite nodules)

Any size

Present with fixed or matted axillary lymph nodes or any infraclavicular, supraclavicular, or internal mammary lymphadenopathy

Ipsilateral supraclavicular lymphadenopathy has been reclassified as regional lymphadenopathy in AJCC $7^{\text {th }}$ edition staging manual ${ }^{[6]}$

AJCC - American Joint Cancer Committee

FNAC is the first diagnostic procedure of choice in breast lesions. Approximately, $73 \%$ cases of TM can be diagnosed by demonstrating epithelioid granulomas with necrosis on cytology ${ }^{[8]}$ Identifying AFB on cytology is particularly difficult in TM due to low bacilli load in such tissues. For AFB to be seen microscopically, their number should be at least $10,000-100,000 / \mathrm{ml}$ in the material. ${ }^{[9]}$ In most cases, classical cytological findings supported by anyone confirmatory test are sufficient for initiating ATT instead of performing invasive biopsy procedures. Histopathological evaluation remains the gold standard. Polymerase chain reaction (PCR) and GeneXpert should be reserved for inconclusive cases. In culture negative paucibacillary forms, PCR is highly sensitive for the diagnosis. Still, the use of PCR is reported only in certain cases for differentiating $\mathrm{TM}$ and other granulomatous mastitis. ${ }^{[10]}$ Other imaging modalities such as mammography provide equivocal results in such cases. Chest imaging is necessary to detect any primary foci in the lung, pleura, or chest. Differentiation from other granulomatous mastitis is of paramount importance because of the implications of corticosteroid therapy in an immunosuppressed patient with tuberculosis.

\section{Conclusions}

FNAC should be the first investigation of choice to rule out malignancy. It saves the patient from other invasive diagnostic procedures like biopsy. In elderly, TM can mimic carcinoma breast. This case thus substantiates the fact that, however rare the entity may be, the clinicians should always keep a broad spectrum of differentials to avoid erroneous diagnosis. The precise diagnosis is must as this helps the clinicians to implement the specific treatment, thereby improving the patient survival.

\section{Declaration of patient consent}

The authors certify that they have obtained all appropriate patient consent forms. In the form the patient(s) has/have given his/her/their consent for his/her/their images and other clinical information to be reported in the journal. The patients understand that their names and initials will not be published and due efforts will be made to conceal their identity, but anonymity cannot be guaranteed.

\section{Financial support and sponsorship}

Nil.

\section{Conflicts of interest}

There are no conflicts of interest.

\section{References}

1. Sabageh D, Amao EA, Ayo-Aderibigbe AA, Sabageh AO. Tuberculous mastitis simulating carcinoma of the breast in a young Nigerian woman: A case report. Pan Afr Med J 2015;21:125.

2. Hamit HF, Ragsdale TH. Mammary tuberculosis. J R Soc Med 1982;75:764-5.

3. Sriram K, Moffatt D, Stapledon R. Tuberculosis infection of the breast mistaken for granulomatous mastitis: A case report. Cases J 2008;1:273.

4. Tewari M, Shukla HS. Breast tuberculosis: Diagnosis, clinical features and management. Indian $\mathrm{J}$ Med Res 2005;122:103-10.

5. Shukla HS, Kumar S. Benign breast disorders in nonwestern populations: Part II - Benign breast disorders in India. World J Surg 1989;13:746-9.

6. Garg PK, Prakash G. Current definition of locally advanced breast cancer. Curr Oncol 2015;22:e409-10.

7. Yamauchi H, Woodward WA, Valero V, Alvarez RH, Lucci A, Buchholz TA, et al. Inflammatory breast cancer: What we know and what we need to learn. Oncologist 2012;17:891-9.

8. Kakkar S, Kapila K, Singh MK, Verma K. Tuberculosis of the breast. A cytomorphologic study. Acta Cytol 2000;44:292-6.

9. Pagel W, Simmonds FA, Macdonald J, Nassan E. Pulmonary Tuberculosis. $4^{\text {th }}$ ed. London: Oxford University Press; 1964. p. 245.

10. Tse GM, Poon CS, Ramachandram K, Ma TK, Pang LM, Law BK, et al. Granulomatous mastitis: A clinicopathological review of 26 cases. Pathology 2004;36:254-7. 